\title{
Medication Adherence and Healthcare Costs Among Patients with Pulmonary Arterial Hypertension Treated with Oral Prostacyclins: A Retrospective Cohort Study
}

\author{
Bonnie B. Dean ${ }^{1} \cdot$ Vishal Saundankar $^{1} \cdot$ Dana Stafkey-Mailey $^{1} \cdot$ Rebekah H. Anguiano $^{2} \cdot$ Andrew C. Nelsen $^{3}$. \\ Kathryn Gordon ${ }^{3} \cdot$ Peter Classi ${ }^{3}$
}

Published online: 6 March 2020

(c) The Author(s) 2020

\begin{abstract}
Background Given the improved convenience of oral prostacyclins, there is a shift toward their use in treating pulmonary arterial hypertension (PAH).

Objectives Our objective was to compare patient characteristics, medication adherence, healthcare resource use (HCRU), and costs among patients receiving oral treprostinil or selexipag.

Methods We used Truven Health MarketScan Commercial and Medicare databases to identify patients with PAH with a diagnosis code for pulmonary hypertension (PH) plus a prescription for oral treprostinil or selexipag from July 2013 to September 2017. Medication adherence, persistence, and all-cause and PAH-related HCRU and costs were compared between cohorts during the 6-month follow-up. Adjusted healthcare costs were obtained using recycled predictions and bootstrapped samples. Results A total of 256 (130 oral treprostinil, 126 selexipag) patients fulfilled the study criteria. The oral treprostinil cohort was more likely to be male, to have previously used parenteral prostacyclins, and to have higher outpatient costs at baseline than the selexipag cohort. During follow-up, both cohorts had similar proportions of patients who were adherent to and persistent with their respective therapies. All-cause and PAH-related medical utilization was generally similar between cohorts. The oral treprostinil cohort had $66.9 \%$ lower total PAH-related healthcare costs (mean difference $-\$ 75,183$; $95 \%$ confidence interval [CI] - 102,584 to - 49,771) and 70.6\% lower PAH-related pharmacy costs (mean difference $-\$ 76,439 ; 95 \%$ CI - 104,512 to - 51,458) than the selexipag cohort, with similar differences in all-cause healthcare and pharmacy costs.

Conclusions Lower all-cause and PAH-related total healthcare and pharmacy costs were observed in patients receiving oral treprostinil compared with those receiving selexipag. It will be important to study longer-term costs and clinical outcomes.
\end{abstract}

\section{Introduction}

Pulmonary arterial hypertension (PAH) is a chronic disease characterized by increased pulmonary vascular resistance and progressive right ventricular dysfunction [1]. Survival in patients with PAH is poor but has improved considerably in the era since the US FDA's approval of therapies indicated for PAH [2]. The 5-year survival in patients with PAH

Bonnie B. Dean

bonnie.dean@xcenda.com

Xcenda, LLC, Palm Harbor, FL, USA

2 College of Pharmacy, University of Illinois at Chicago, Chicago, IL, USA

3 United Therapeutics Corporation, Research Triangle Park, NC, USA ranges from 59 to $68 \%$ [3-5]. Various risk score calculators have been developed for predicting contemporary survival [6-9], and certain indicators, including B-type natriuretic peptide, $\mathrm{N}$-terminal pro $\mathrm{B}$-type natriuretic peptide, functional status, and 6-minute walk distance, are predictors of outcomes and survival $[5,10,11]$.

Treatment guidelines have historically recommended initial monotherapy determined by baseline severity of World Health Organization (WHO) functional class (FC) with PAH-approved therapies from four medication classes, including endothelin receptor agonists (ERA), phosphodiesterase-5 inhibitors (PDE-5i), prostacyclin-class therapies, and soluble guanylate cyclase (SGC) stimulators, followed by sequential combination therapy for patients in whom response is inadequate [12]. Following results from the AMBITION trial and other research showing a lower risk of clinical failure events and improved survival in patients 


\section{Key Points}

There is a shift toward the use of oral prostacyclins in treating pulmonary arterial hypertension (PAH) given the improved convenience of this administration route relative to parenteral and inhaled therapy.

Real-world claims data show relatively similar utilization patterns, with lower all-cause and PAH-related total healthcare and pharmacy costs observed in patients receiving oral treprostinil compared with selexipag.

with upfront combination therapy [13, 14], clinical practice guidelines have increasingly recommended initial combination drug therapy $[1,15]$.

Prostacyclins are a member of the eicosanoid family of lipids produced from arachidonic acid in a multistep process. The binding of prostacyclin to platelets and endothelial cells results in the inhibition of platelet aggregation, relaxation of smooth muscle, and pulmonary artery dilation, which mediates the vasoconstriction associated with PAH and improves cardiac output [16]. Prostacyclin-class therapies are most often used in combination therapy in either first- or secondline regimens in patients with advanced FC [1, 17] and are prescribed to about one-quarter of patients [17-19]. More recently, there has been a shift from parenteral to nonparenteral prostacyclin use [19]. Treprostinil extended-release tablets and selexipag are two oral therapies that target the prostacyclin pathway and are approved for the treatment of PAH. Since the FDA approval of oral treprostinil in 2013 and selexipag in 2015, which have improved convenience and safety compared with parenteral prostanoids, use of oral prostacyclin therapies has increased and is likely to continue [17, 19].

The goal of treatment is to reduce the progression of symptoms, improve FC [20, 21], and lower mortality risk among patients with PAH [6-9]. Recent studies have shown that oral prostacyclin therapies may be effective at delaying disease progression and reducing the risk of mortality [22, 23]. Results of the FREEDOM-EV study of oral treprostinil in patients with PDE-5i/SGC stimulator or ERA background monotherapy reported a clinically and statistically significant reduction in clinical worsening, defined as death from any cause, hospitalization for worsening PAH, disease progression, initiation of inhaled or infused prostacyclin therapy, or unsatisfactory long-term clinical response [24]. Similarly, the GRIPHON study of selexipag in patients with either no background therapy, a PDE-5i, and/or an ERA demonstrated a reduction in clinical worsening, defined as death from any cause or a PAH-related complication, including disease progression or worsening that resulted in hospitalization, initiation of parenteral prostanoid therapy or long-term oxygen therapy, or the need for lung transplantation or balloon atrial septostomy [21].

While the safety and efficacy of oral prostacyclin therapies is well-established, little is known about their impact on the utilization and costs of PAH-related care in the real-world setting, as reduced costs can reflect an important benefit of treatment. This study identified patients initiating oral treprostinil versus selexipag using claims data and evaluated realworld differences in patient characteristics, medication adherence, persistence, healthcare resource use (HCRU), and costs.

\section{Methods}

\subsection{Study Design and Data Source}

This retrospective cohort study used integrated healthcare claims data from the IBM $^{\circledR}$ MarketScan databases (MarketScan), including the Commercial Claims Encounters Database and Medicare Supplemental Database during the study period from 1 July 2013 to 30 September 2017 (Fig. 1). Adults with PAH aged $\geq 18$ years were identified using a recommended algorithm for identifying patients with PAH from claims data, which required a diagnosis code for pulmonary hypertension $(\mathrm{PH})$ plus a prescription for oral treprostinil or selexipag [25]. PH diagnosis was identified by International Classification of Diseases, Ninth Revision, Clinical Modification (ICD-9-CM) and ICD-10-CM diagnostic codes (ICD-9-CM: 416.0, 416.8, 416.9; ICD-10-CM: I27.0, I27.2, I27.81, I27.89, I27.9). Oral prostacyclin therapies were identified using national drug codes available within the pharmacy claims. The index date was the date of the first prescription for oral prostacyclin therapy during the identification period from 1 January 2014 to 31 March 2017.

Patients were required to have had continuous enrollment in the health plan during the 6-month pre-index and postindex periods, a $\mathrm{PH}$ diagnosis code during the 6-month preindex period, and no pharmacy claims for oral treprostinil or selexipag during the pre-index period. Baseline demographic and clinical characteristics and utilization were assessed in the 6-month pre-index period, and HCRU, costs, and treatment patterns, including adherence, persistence, and dosing, were assessed during the 6-month post-index period. Healthcare Common Procedure Coding System codes were used for identifying medical claims for parenteral prostacyclin-class therapies.

\subsection{Study Outcomes}

Treatment adherence was measured using the medication possession ratio (MPR) and the proportion of days covered 


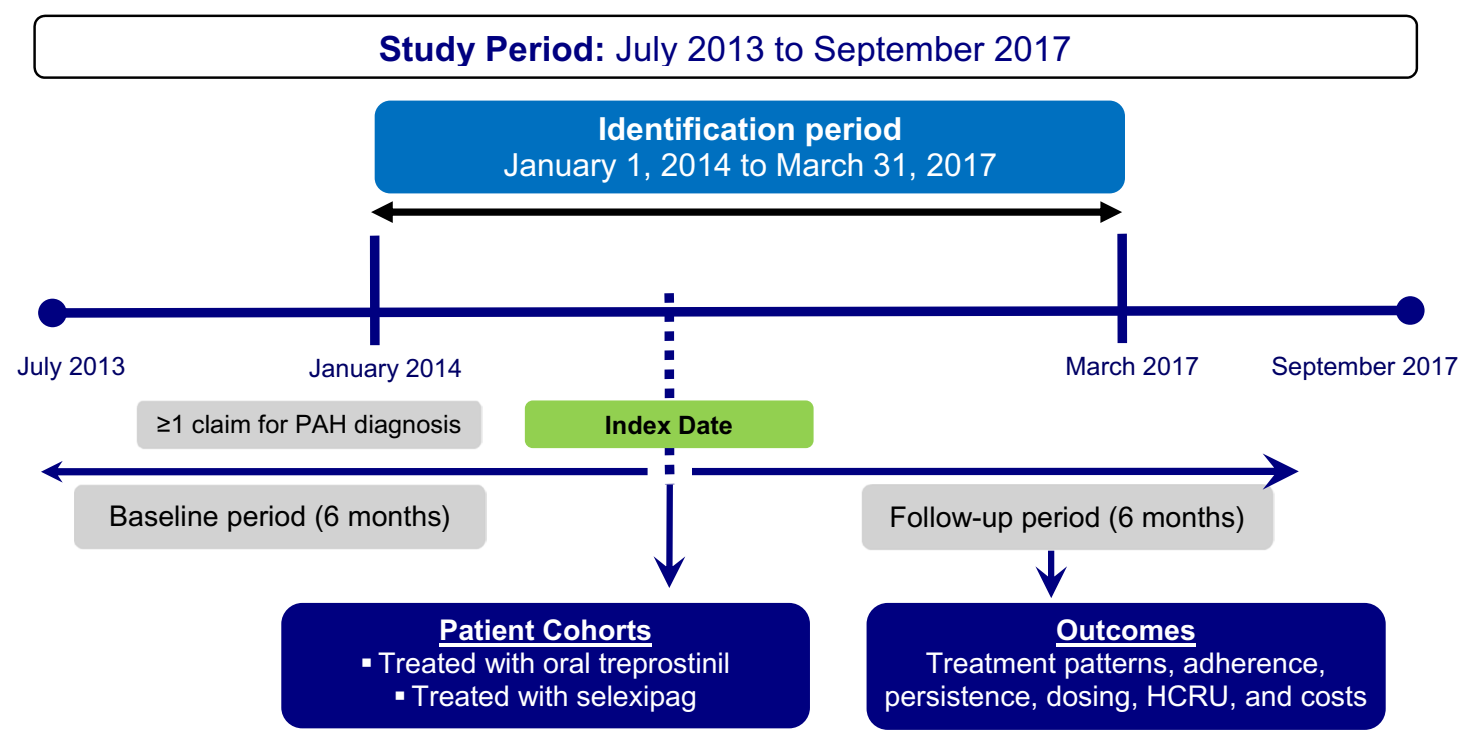

Fig. 1 Study design. The index date is defined as first pharmacy claim for oral treprostinil or selexipag. $H C R U$ healthcare resource use, $P A H$ pulmonary arterial hypertension

(PDC). The MPR numerator was defined as the sum of the days of supply for the index regimen medication fills during the 6-month follow-up, and the denominator was the number of days between the first and last fill plus days of supply of the last fill. MPR was reported as a mean percentage ranging from 0 to 1 . MPR was also reported as the proportion of patients with at least $80 \%$ mean MPR. The PDC numerator was defined as the sum of the days of supply for the index regimen medication fills during the 6-month follow-up, and the denominator was the total number of days in the 6-month follow-up. PDC was reported as a mean percentage ranging from 0 to 1 and also reported as the proportion of patients with at least $80 \%$ PDC. Treatment persistence was measured as the proportion of patients who did not have a gap of a defined duration of 60 days in the index therapy regimen during the 6-month follow-up. Time to treatment discontinuation was defined as the number of days of index therapy regimen from the index date to start of the first gap of a defined duration of $\geq 60$ days. A sensitivity analysis was used to assess treatment persistence and time to treatment using a gap of defined duration of $\geq 30$ days.

All-cause and PAH-related HCRU during the baseline and follow-up periods are reported. All-cause HCRU during follow-up was computed by the setting of care identified from the claims data using the place of service variable and applicable Current Procedural Terminology, 4th edition procedure codes. HCRU is reported for medical utilization, broken down by inpatient and outpatient visits, and for pharmacy utilization. Medical utilization was defined as the average number of unique visits per patient by type of visit. Pharmacy utilization was defined as the number of unique prescriptions filled per patient. PAH-related HCRU was identified using PH diagnosis codes in any position.

All-cause and PAH-related healthcare costs during the follow-up period are reported. Costs reflect all payments made to providers of care from both the health plan (plan and coordination of benefits) and the patient (copayment, coinsurance, deductible). All costs were adjusted to \$US, year 2017 values, using the Bureau of Labor Statistics medical care component of the Consumer Price Index.

\subsection{Statistical Analysis}

Descriptive statistics (percentages, means, medians, standard deviations [SDs]) were used to describe baseline demographic/clinical characteristics, adherence and persistence, and dosing for the oral treprostinil and selexipag cohorts. Chi-squared tests or Fischer's exact test were used for comparisons of frequencies between the cohorts, and Wilcoxon-Mann-Whitney tests were used for continuous variables.

Mean doses of oral prostacyclin therapies were calculated for each month of follow-up for patients in each treatment group who were prostacyclin naïve in the 6 months prior to index treatment. For patients receiving selexipag, dosing was calculated following the first claim for a nonstarter pack. Mean doses were evaluated separately in the subset of patients who used a prostacyclin via an alternative administration route prior to oral therapy, as patients transitioning to oral prostacyclin therapies from an alternative route initiate oral treatment at higher daily doses comparable to their daily doses for nonparenteral prostacyclin. A sensitivity analysis was also conducted to explore mean daily doses of 
oral prostacyclin therapies over a longer 12-month period among the subset of patients persistent on oral prostacyclin therapies for 12 months.

Unadjusted HCRU and costs are reported overall and for PAH-related visits as the number and percentage for categorical measures and as means \pm SDs for continuous measures. Adjusted all-cause and PAH-related costs were estimated using recycled predictions and bootstrapped samples [26-28]. Costs during post-index follow-up were adjusted for baseline characteristics, including age, sex, region, and insurance plan type; prior prostacyclin use; presence of comorbid conditions; receipt of right heart catheterization (RHC); and pharmacy, inpatient, and total outpatient costs. Pharmacy costs for oral prostacyclin therapies were also explored in sensitivity analyses among the subset of patients persistent on oral prostacyclin-class therapies for 6 and 12 months. All analyses were conducted using SAS version 9.4 (SAS Institute, Inc; Cary, NC, USA).

\section{Results}

A total of 405 patients initiated oral treprostinil or selexipag during the study period. After applying study criteria, 256 patients with PAH (130 receiving oral treprostinil; 126 receiving selexipag) met the study criteria. Table 1 provides a description of the baseline demographic and clinical characteristics of the study cohort. Oral treprostinil and selexipag patients were of similar age (mean $58.0 \pm 16.2$ vs. $58.5 \pm 13.0$ years for oral treprostinil and selexipag, respectively) and had a similar Charlson Comorbidity Index (mean $3.5 \pm 2.6$ vs. $3.2 \pm 2.2$ for oral treprostinil and selexipag, respectively). The proportion of males was higher in the oral treprostinil cohort (33.1 vs. $16.7 \%$ for oral treprostinil and selexipag, respectively; $p<0.01)$. Rates of specific comorbidities varied, with more rheumatic disease in the selexipag cohort (20 vs. $32.5 \%$ for oral treprostinil and selexipag, respectively; $p=0.023$ ) and a nonsignificant trend toward more renal disease and AIDS/HIV in the oral treprostinil cohort. There was a $10 \%$ difference in the proportion of patients who underwent RHC in the 6 months prior to being prescribed oral prostacyclin, although this difference did not meet statistical significance ( 47.7 vs. $36.5 \%$ for oral treprostinil and selexipag, respectively; $p=0.07$ ).

Baseline treatment patterns were compared between the cohorts (Table 1). A higher proportion of patients in the selexipag cohort used a PDE-5i alone or in combination with an ERA (73.8 vs. $66.2 \%$ for selexipag and oral treprostinil, respectively; $p=0.18$ ) during baseline, although this difference was not statistically significant. More patients receiving oral treprostinil transitioned from a parenteral prostacyclin (14.6 vs. $4.0 \%$ for oral treprostinil and selexipag, respectively; $p=0.004)$.
Baseline total all-cause costs were similar between the cohorts except for all-cause outpatient costs, which were higher in the oral treprostinil cohort than in the selexipag cohort (mean $\$ 23,883 \pm 88,718$ and $\$ 11,600 \pm 17,450$, respectively; $p=0.025$ ) (Table 1 ). Mean baseline PAHrelated medical and pharmacy costs were similar across the cohorts, as were PAH-related total healthcare costs.

\subsection{Adherence and Persistence}

Following the initiation of oral prostacyclin therapy, the mean MPR was higher for oral treprostinil than for selexipag $(0.89 \pm 0.19$ vs. $0.85 \pm 0.22$, respectively; $p=0.039)$. While the proportion of patients with MPR $\geq 80$ was higher in the oral treprostinil cohort $(82 \%)$ than in the selexipag cohort $(77 \%)$, this difference was not statistically significant (Fig. 2). Mean PDC was similar between the cohorts $(0.72 \pm 0.31$ vs. $0.71 \pm 0.31$ for oral treprostinil and selexipag, respectively; $p=0.483$ ), and the proportion of patients with PDC $\geq 80$ was the same in both cohorts $(56 \%$; $p=0.975$ ). There was no significant difference between the cohorts in the proportion of patients persistent on therapy when discontinuation was defined as a gap of $\geq 60$ days (71 vs. $72 \%$ for oral treprostinil and selexipag, respectively; $p=0.797$ ) or a gap of $\geq 30$ days (62 vs. $63 \%$, respectively; $p=0.797)$.

\subsection{Prescriptions and Monthly Dosing}

The mean number of prescriptions was $6.1 \pm 3.3$ and $5.8 \pm 2.7$ for oral treprostinil and selexipag, respectively, which did not differ between cohorts $(p=0.310)$. Similarly, the median and interquartile range (IQR) for the number of prescriptions was $6.5(4-7)$ and $6(5-7)$ for oral treprostinil and selexipag, respectively.

In prostacyclin-naïve patients, the median total daily dose at 6 months was $6.9 \mathrm{mg}$ (IQR 2.2-9.5) for oral treprostinil and $2213 \mu \mathrm{g}$ (IQR 1200-3093) for selexipag. Median total daily dose generally increased from month 1 through 6 . In sensitivity analysis over 12 months post-index, daily median doses generally reached their highest point in month 6 and did not fluctuate much from 6 through 12 months.

In patients who received parenteral prostacyclin before initiation of oral prostacyclin, median total daily doses started higher, as expected, but also remained higher over 6-month follow-up than in those who were prostacyclin naïve. Among patients who received prior parenteral prostacyclin-class therapies, the median total daily dose at 6 months was $11.8 \mathrm{mg}$ (IQR 4.4-20.4) for oral treprostinil and $2600 \mu \mathrm{g}$ (IQR 1365-3200) for selexipag. In a sensitivity analysis over the 12-month post-index period among these patients, median total daily doses reached their highest points between months 6 and 12 for both cohorts, generally 
Table 1 Baseline demographic, clinical characteristics, treatments, and costs

\begin{tabular}{|c|c|c|c|}
\hline Characteristics, treatments, and costs & Oral treprostinil $(N=130)$ & Selexipag $(N=126)$ & $p$ value \\
\hline \multicolumn{4}{|l|}{ Demographic characteristics } \\
\hline Age, years & $58.0 \pm 16.2$ & $58.5 \pm 13.0$ & 0.755 \\
\hline Males & $43(33.1)$ & $21(16.7)$ & 0.002 \\
\hline Geographic region & & & 0.106 \\
\hline Northeast & $31(23.9)$ & $16(12.7)$ & \\
\hline North Central & $31(23.9)$ & $30(23.8)$ & \\
\hline South & $54(41.5)$ & $60(47.6)$ & \\
\hline West & $14(10.8)$ & $20(15.9)$ & \\
\hline Payer type & & & 0.139 \\
\hline Commercial & $86(66.2)$ & 94 (74.6) & \\
\hline Medicare supplemental & $44(33.9)$ & $32(25.4)$ & \\
\hline \multicolumn{4}{|l|}{ Clinical characteristics } \\
\hline CCI, mean (SD) & $3.5 \pm 2.6$ & $3.2 \pm 2.2$ & 0.485 \\
\hline \multicolumn{4}{|l|}{ CCI comorbidities } \\
\hline Myocardial infarction & $5(3.9)$ & $1(0.8)$ & 0.214 \\
\hline Congestive heart failure & $64(49.2)$ & $54(42.9)$ & 0.306 \\
\hline Peripheral vascular disease & $18(13.9)$ & $19(15.1)$ & 0.779 \\
\hline Cerebrovascular disease & $9(6.9)$ & $5(4.0)$ & 0.412 \\
\hline Dementia & $0(1.0)$ & $2(3.0)$ & - \\
\hline Chronic pulmonary disease & $127(97.7)$ & $126(100.0)$ & 0.247 \\
\hline Rheumatic disease & $26(20.0)$ & $41(32.5)$ & 0.023 \\
\hline Peptic ulcer disease & $0(0.0)$ & $1(0.8)$ & 0.492 \\
\hline Mild liver disease & $8(6.2)$ & $5(4.0)$ & 0.572 \\
\hline Diabetes without chronic complication & $32(24.6)$ & $32(25.4)$ & 0.885 \\
\hline Diabetes with chronic complication & $17(13.1)$ & $26(20.6)$ & 0.106 \\
\hline Hemiplegia/paraplegia & $0(1.0)$ & $2(3.0)$ & - \\
\hline Renal disease & $29(22.3)$ & $17(13.5)$ & 0.066 \\
\hline Malignancy (any) & $11(8.5)$ & $7(5.6)$ & 0.363 \\
\hline Moderate or severe liver disease & $4(3.1)$ & $5(4.0)$ & 0.746 \\
\hline AIDS/HIV & $5(3.9)$ & $0(0.0)$ & 0.060 \\
\hline Right heart catheterization & $62(47.7)$ & $46(36.5)$ & 0.070 \\
\hline Echocardiography & $88(67.7)$ & $85(67.5)$ & 0.968 \\
\hline \multicolumn{4}{|l|}{ Baseline PAH treatment classes } \\
\hline ERAs & $94(72.3)$ & $96(76.2)$ & 0.478 \\
\hline PDE-5is & $86(66.2)$ & $93(73.8)$ & 0.182 \\
\hline sGC stimulator & $15(11.5)$ & $16(12.7)$ & 0.776 \\
\hline ERA + PDE-5i combination therapy & $57(43.9)$ & $65(51.6)$ & 0.215 \\
\hline $\mathrm{ERA}+\mathrm{sGC}$ combination therapy & $9(6.9)$ & $11(8.7)$ & 0.590 \\
\hline Intravenous treprostinil & $17(13.1)$ & $3(2.4)$ & 0.002 \\
\hline Inhaled treprostinil & $23(17.7)$ & $24(19.1)$ & 0.779 \\
\hline Epoprostenol & $3(2.3)$ & $2(1.6)$ & 1.000 \\
\hline Iloprost & $5(3.9)$ & $5(4.0)$ & 1.000 \\
\hline $\begin{array}{l}\text { Any parenteral prostacyclin (intravenous trepro- } \\
\text { stinil, epoprostenol) }\end{array}$ & $19(14.6)$ & $5(4.0)$ & 0.004 \\
\hline \multicolumn{4}{|l|}{ Healthcare costs } \\
\hline Total healthcare cost & $\$ 105,249 \pm 113,376$ & $\$ 93,619 \pm 95,309$ & 0.360 \\
\hline Medical cost & $\$ 34,096 \pm 100,989$ & $\$ 18,288 \pm 25,384$ & 0.054 \\
\hline Inpatient cost & $\$ 10,212 \pm 37,226$ & $\$ 6688 \pm 18,038$ & 0.389 \\
\hline Total outpatient cost & $\$ 23,883 \pm 88,718$ & $\$ 11,600 \pm 17,450$ & 0.025 \\
\hline Prescription costs & $\$ 71,153 \pm 59,347$ & $\$ 75,331 \pm 95,647$ & 0.999 \\
\hline
\end{tabular}

Data are presented as mean $\pm \mathrm{SD}$ or $\mathrm{N}(\%)$ unless otherwise indicated. Costs are presented as $\$$ US, year 2017 values

AIDS acquired immunodeficiency syndrome, $C C I$ Charlson comorbidity index, ERA endothelin receptor antagonist, $H I V$ human immunodeficiency virus, $P A H$ pulmonary arterial hypertension, $P D E-5 i$ phosphodiesterase type-5 inhibitor, $S D$ standard deviation, $s G C$ soluble guanylate cyclase 
Fig. 2 Medication adherence and persistence. $M P R$ medication possession ratio, $P D C$ proportion of days covered. $* p<0.05$

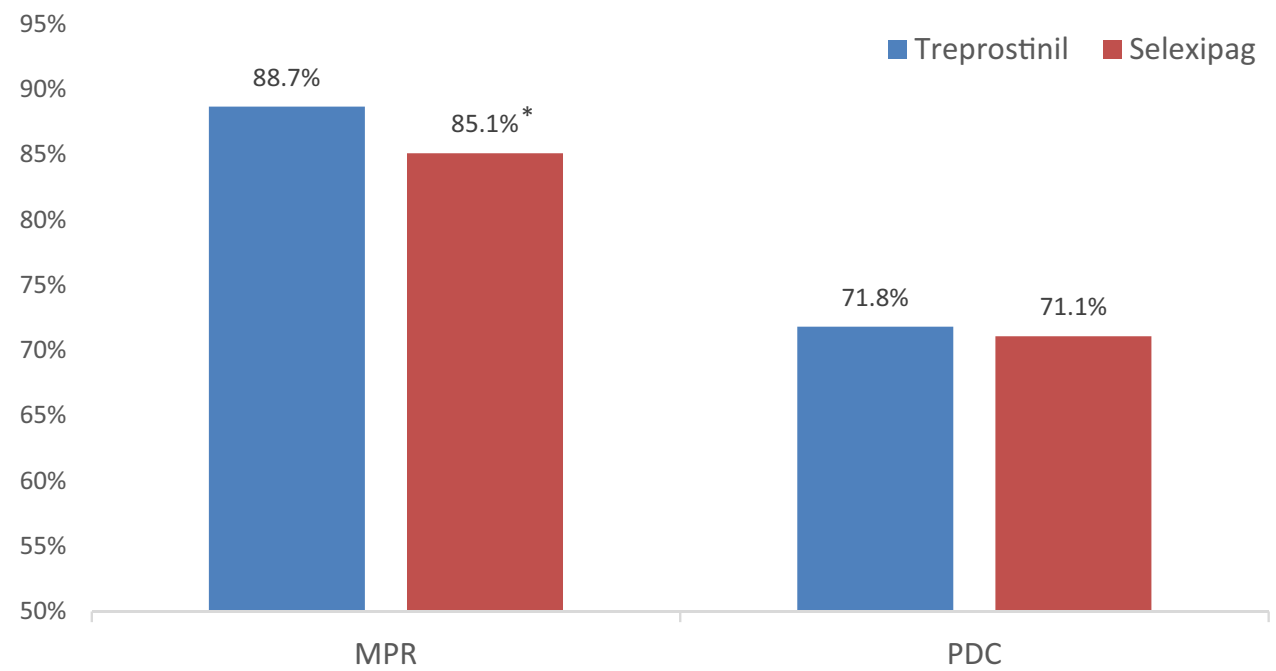

later than for those who were prostacyclin naïve at the time of oral prostacyclin initiation.

\subsection{Healthcare Resource Use and Costs}

There was no significant difference between the cohorts in all-cause inpatient visits (Table 2) during the post-index period. The adjusted mean number of inpatient visits was $1.3 \pm 0.6$ for the oral treprostinil cohort and $1.9 \pm 0.8$ for the selexipag cohort $(p=0.199)$. Adjusted mean length of hospital stay was $8.6 \pm 6.1$ days for the oral treprostinil cohort and $10.4 \pm 7.3$ days for the selexipag cohort $(p=0.441)$. Similarly, the mean number of all-cause emergency department (ED) visits was similar $(0.4 \pm 1.1$ and $0.4 \pm 1.0$ for oral treprostinil and selexipag, respectively; $p=0.917)$. However, the mean all-cause adjusted outpatient visits was higher in the oral treprostinil cohort $(22.0 \pm 8.4$ vs. $18.8 \pm 7.2$ for oral treprostinil and selexipag, respectively; $p<0.001$ ).

There was no significant difference between the two cohorts for PAH-related medical utilization (inpatient visits, ED visits, and outpatient visits) or the number of unique PAH-related prescriptions (Table 2). The mean number of all-cause unique prescriptions was similar between the cohorts $(45 \pm 20$ vs. $49 \pm 22$ for oral treprostinil and selexipag, respectively; $p=0.121$ ). Notably, a higher proportion of patients in the oral treprostinil cohort received RHC during the post-index period compared with the selexipag cohort (13.2 vs. $5.6 \%$, respectively; $p=0.039$ ).

All-cause healthcare costs differed between the cohorts (Fig. 3). The mean all-cause total pharmacy cost during the follow-up was higher in the cohort treated with selexipag $(\$ 171,169 \pm 111,812)$ than in the cohort treated with oral treprostinil $(\$ 118,962 \pm 110,675 ; p<0.001)$, and this largely contributed to the difference in total costs $(\$ 189,375 \pm 114,549)$ compared with the cohort treated with oral treprostinil $(\$ 153,457 \pm 159,761 ; p<0.001)$. Similar results were seen when comparing PAH-related total healthcare and pharmacy costs.

A sensitivity analysis was also conducted to explore pharmacy differences among persistent users throughout 6 and 12 months of follow-up (Table 3). Analysis conducted over 6 and 12 months showed that higher pharmacy costs persisted in the selexipag cohort compared with the oral treprostinil cohort. At 6 months, the median pharmacy costs directly attributable to oral treprostinil (\$39,392 [IQR 21,483-77,748]) were significantly less than for selexipag (\$127,335 [IQR 109,908-136,030]; $p<0.0001)$. Similarly, median pharmacy costs for persistent users over 12 months directly attributable to oral treprostinil (\$125,420 [IQR 60,043-194,605]) were significantly less than for selexipag (\$219,250 [IQR 201,624-239,696]; $p<0.0001)$.

After adjustment for covariates, treatment with oral treprostinil was associated with $51.4 \%$ lower total all-cause healthcare costs than treatment with selexipag (difference in mean - \$73,680; 95\% confidence interval [CI] - 103,702 to $-43,963$ ) (Fig. 4). This difference in all-cause healthcare costs was largely driven by all-cause pharmacy costs, which were $68.2 \%$ lower in patients being treated with oral treprostinil than in those being treated with selexipag (difference in mean - \$77,654; 95\% CI - 105,392 to - 52,118). Treatment with oral treprostinil was associated with $66.9 \%$ lower total PAH-related healthcare costs compared with being treated with selexipag (difference in mean - \$75,183; 95\% CI - 102,584 to - 49,771). Differences in PAH-related healthcare costs were largely driven by PAH-related pharmacy costs, which were $70.62 \%$ lower in patients treated with oral treprostinil than in those treated with selexipag (difference in mean $-\$ 76,439 ; 95 \% \mathrm{CI}-104,512$ to $-51,458)$. 
Table 2 Healthcare resource utilization during the post-index period

\begin{tabular}{|c|c|c|c|}
\hline Healthcare resource utilization & $\begin{array}{l}\text { Oral treprostinil } \\
(N=130)\end{array}$ & $\begin{array}{l}\text { Selexipag } \\
(N=126)\end{array}$ & $p$ value \\
\hline \multicolumn{4}{|c|}{ All-cause healthcare resource utilization } \\
\hline Inpatient visits & $43(33.1)$ & $29(23.0)$ & \\
\hline Inpatient visits ${ }^{\mathrm{a}}$ & $0.4 \pm 0.7$ & $0.4 \pm 0.9$ & 0.127 \\
\hline Adjusted inpatient visits ${ }^{\mathrm{b}}$ & $1.3 \pm 0.6$ & $1.9 \pm 0.8$ & 0.199 \\
\hline Length of stay per hospitalization & $5.8 \pm 7.3$ & $7.1 \pm 7.4$ & 0.297 \\
\hline Adjusted length of stay ${ }^{\mathrm{b}}$ & $8.6 \pm 6.1$ & $10.4 \pm 7.3$ & 0.441 \\
\hline Bed days $^{\mathrm{a}}$ & $2.5 \pm 6.4$ & $2.8 \pm 7.3$ & 0.156 \\
\hline \multicolumn{4}{|l|}{ Outpatient visits } \\
\hline ED visits & $33(25.4)$ & $32(25.4)$ & 0.998 \\
\hline ED visits ${ }^{\mathrm{a}}$ & $0.4 \pm 1.1$ & $0.4 \pm 1.0$ & 0.917 \\
\hline Overall outpatient visits & $130(100.0)$ & $126(100.0)$ & - \\
\hline Overall outpatient visits ${ }^{\mathrm{a}}$ & $22.1 \pm 16.6$ & $18.7 \pm 10.0$ & 0.258 \\
\hline Adjusted overall outpatient visits ${ }^{b}$ & $22.0 \pm 8.4$ & $18.8 \pm 7.2$ & $<0.0001$ \\
\hline Unique prescriptions & $45 \pm 20$ & $49 \pm 22$ & 0.121 \\
\hline \multicolumn{4}{|c|}{ PAH-related healthcare resource utilization } \\
\hline Inpatient visits & $10(7.7)$ & $5(4.0)$ & 0.205 \\
\hline Inpatient visits $^{\mathrm{a}}$ & $0.1 \pm 0.3$ & $0.1 \pm 0.3$ & 0.211 \\
\hline Adjusted inpatient visits ${ }^{\mathrm{b}}$ & $1.2 \pm 0.6$ & $1.2 \pm 0.6$ & 1.000 \\
\hline Length of stay per hospitalization & $4.0 \pm 2.3$ & $5.0 \pm 6.3$ & 0.673 \\
\hline Adjusted length of stay ${ }^{\mathrm{b}}$ & $1.2 \pm 0.4$ & $1.3 \pm 0.4$ & 0.397 \\
\hline Bed days $^{\mathrm{a}}$ & $0.3 \pm 1.4$ & $0.3 \pm 2.0$ & 0.201 \\
\hline \multicolumn{4}{|l|}{ Outpatient visits } \\
\hline ED visits & $3(2.3)$ & $0(0.0)$ & 0.086 \\
\hline ED visits $^{\mathrm{a}}$ & $0.04 \pm 0.3$ & $0.0 \pm 0.0$ & 0.088 \\
\hline Overall outpatient visits & $125(96.2)$ & $119(94.4)$ & 0.518 \\
\hline Overall outpatient visits ${ }^{\mathrm{a}}$ & $5.8 \pm 4.8$ & $5.4 \pm 4.8$ & 0.280 \\
\hline Adjusted overall outpatient visits ${ }^{\mathrm{b}}$ & $5.8 \pm 2.17$ & $6.0 \pm 2.21$ & 0.748 \\
\hline PAH-specific unique prescriptions & $13.9 \pm 6.4$ & $14 \pm 5.9$ & 0.658 \\
\hline
\end{tabular}

Data are presented as mean $\pm \mathrm{SD}$ or $N(\%)$ unless otherwise indicated

$E D$ emergency department, $P A H$ pulmonary arterial hypertension, $S D$ standard deviation

${ }^{a}$ Mean \pm SD were calculated for the entire cohort

${ }^{\mathrm{b}}$ Adjusted for baseline age, sex, region, insurance plan type, prior prostacyclin use, presence of comorbid conditions, receipt of RHC and respective baseline pharmacy, inpatient, and total outpatient costs

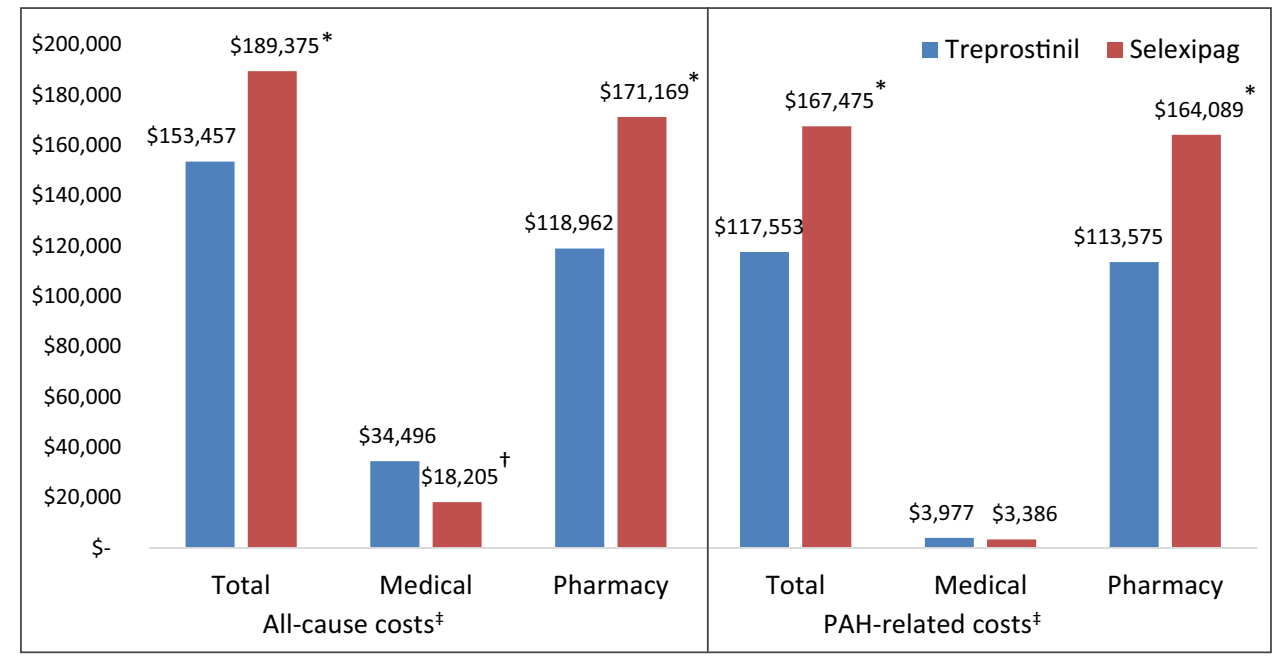

Fig. 3 Unadjusted all-cause and PAH-related healthcare costs. $* P<0.001,{ }^{\dagger} P<0.05,{ }^{\ddagger} \$ \mathrm{US}$, year 2017 values. $P A H$ pulmonary arterial hypertension 


\section{Discussion}

This is the first claims database study to compare treatment patterns and outcomes for patients with PAH initiating therapy with oral treprostinil versus selexipag. Results from this real-world study suggest there were no significant differences in all-cause and PAH-related medical HCRU between patients with PAH initiating therapy with oral treprostinil or selexipag during follow-up, except for all-cause outpatient visits, but there were significant differences in pharmacy utilization. Results suggest that treatment with oral treprostinil was associated with 51.4\% lower total all-cause healthcare costs compared with treatment with selexipag, and this was largely driven by all-cause pharmacy costs, which were $68.2 \%$ lower in patients treated with oral treprostinil than in those treated with selexipag. Treatment with oral treprostinil was associated with $66.9 \%$ lower total PAH-related healthcare costs compared with treatment with selexipag, again largely driven by $70.6 \%$ lower PAH-related pharmacy costs in patients being treated with oral treprostinil than in those being treated with selexipag.

Medical care utilization was similar between the study cohorts receiving oral prostacyclin therapies. Notably, both cohorts reported similar hospitalization rates in both unadjusted and adjusted analyses. All-cause inpatient visits were comparable to those reported by Burger et al. [19], in which the mean number of inpatient visits in the 6 months following the initiation of nonparenteral prostacyclin therapies was also 0.4. However, mean PAH-related inpatient visits were lower in our study cohorts ( 0.1 for both oral treprostinil and selexipag cohorts) than the 0.3 reported by Burger et al. [19]. While the all-cause physician office visits were higher in the oral treprostinil cohort, PAH-related visits were similar between the oral prostacyclin cohorts. More physician office visits could be attributed to a higher proportion of patients in the oral treprostinil cohort receiving prior parenteral prostacyclin, which requires more frequent medical visits and may reflect a more advanced PAH disease stage. However, measures of PAH disease severity, such as FC and 6-minute walk distance, which may be useful for adjusting baseline differences, are not available within claims data. In the 6 months following oral prostacyclin initiation, a greater proportion of the oral treprostinil cohort received RHC. Taken together, these suggest that patients in the oral treprostinil cohort may have been sicker and hence more closely managed by their providers. However, it is important to point out that PAH-related medical costs decreased in both cohorts following the initiation of oral prostacyclin, and this reflects an important benefit of successful treatment. Our
Table 3 Cost of oral treprostinil vs. selexipag among patients persistent on therapy

\begin{tabular}{llll}
\hline Oral prostacyclin costs & Oral treprostinil & Selexipag & $p$ value \\
\hline Patients persistent for 6 months & $N=92$ & $N=91$ & \\
Mean \pm SD & $69,745 \pm 106,688$ & $136,494 \pm 81,582$ & \\
Median (IQR) & $39,392(21,483-77,748)$ & $127,335(109,908-136,030)$ & $<0.0001$ \\
Patients persistent for 12 months & $N=52$ & $N=47$ & \\
Mean \pm SD & $189,222 \pm 258,543$ & $230,852 \pm 102,717$ & \\
Median (IQR) & $125,420(60,043-194,605)$ & $219,250(201,624-239,696)$ & $<0.0001$ \\
\hline
\end{tabular}

Costs are presented as \$US, year 2017 values

$I Q R$ interquartile range, $S D$ standard deviation

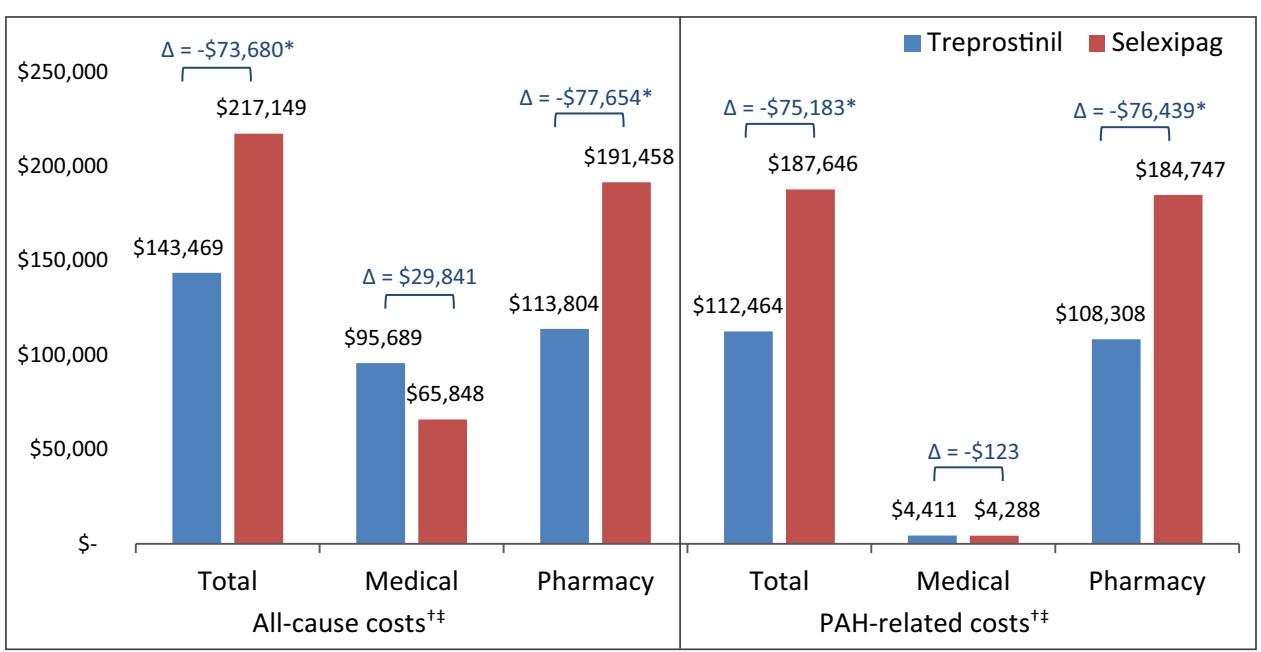

Fig. 4 Adjusted all-cause and PAH-related healthcare costs. $* P<0.05$. ${ }^{\dagger}$ Adjusted for baseline age, sex, region, insurance plan type, prior prostacyclin use, presence of comorbid conditions, and receipt of RHC and baseline pharmacy, inpatient, and total outpatient costs. $\$$ US, year 2017 values. $P A H$ pulmonary arterial hypertension, $R H C$ right heart catheterization 
study shows that most of the healthcare cost was composed of pharmacy costs in both cohorts. This observation is similar to that in the study by Burger et al. [19], who reported that mean all-cause pharmacy costs in patients treated with nonparenteral prostacyclins therapies for 6 months were $\$ 96,539 \pm 49,770$, representing about $75 \%$ of the mean total all-cause healthcare costs of $\$ 128,427 \pm 120,938$.

These real-world data also suggest that medication adherence and persistence with oral prostacyclin therapies were similar in the cohorts. MPR to oral prostacyclin was high in both the oral treprostinil (89\%) and selexipag (85\%) cohorts, similar to that reported by Burger et al. (89\%) for prostacyclin use [18]. However, PDC from this study (72 and 71\% for oral treprostinil and selexipag, respectively) were lower than those reported by Studer et al. [17] when prostacyclin therapies were initiated as part of combination therapy (90\%), but the estimates from this study were higher than when prostacyclin therapies were initiated as monotherapy (50\%).

Median total daily dosing for both oral prostacyclinclass therapies increased during the 6-month follow-up but generally remained at the same level through 12 months in patients with persistent use, suggesting that dosing titration usually takes up to 6 months following initiation of oral prostacyclin therapies. However, in patients transitioning from parenteral to oral prostacyclin, median total daily doses at initiation and throughout 12 months were higher than in prostacyclin-naïve patients. Those transitioning reached their highest levels between 6 and 12 months, peaking higher than in prostacyclin-naïve patients. This finding may reflect differences in patient severity, oral prostacyclin tolerance, or medical management. It should be noted that prior prostacyclin use at baseline was, therefore, controlled for in the analysis of HCRU and costs. Importantly, the transition from parenteral therapy was more common in those receiving oral treprostinil, and-after controlling for this-they still had lower healthcare costs than those in the selexipag cohort.

The difference in cost for oral prostacyclins cannot be directly attributable to variations in the wholesale acquisition cost of the respective drugs. The pricing models for oral treprostinil and selexipag are not directly comparable, as oral treprostinil is based on a price-per-dose model, with increased pricing for higher-dosage tablets, whereas selexipag is based on a fixed price that does not vary by dose. While fixed pricing, regardless of dosage, is often perceived as less costly and predictable [29], dose-based pricing can result in lower costs with dose reductions [30]. This study showed that, for oral prostacyclin drugs used in patients with $\mathrm{PAH}$, treprostinil dose-based pricing led to lower pharmacy costs than the fixed pricing associated with selexipag.

Given that all HCRU, except for the number of outpatient visits, were similar between the cohorts during the postindex period and relatively similar at baseline, the higher overall healthcare cost can be attributed to treatment with selexipag. In patients receiving oral treprostinil, the medical cost was slightly higher; however, the difference was not statistically significant and may merely reflect a sicker baseline cohort given that patients receiving oral treprostinil had more often used parenteral therapy and had higher medical costs during baseline than those receiving selexipag. The reduction in medical costs following initiation of therapy in comparison to baseline medical costs reflects an important benefit of oral prostacyclins. Moreover, the slightly higher medical cost in the follow-up period was compensated by the lower overall pharmacy cost among patients receiving oral treprostinil and a larger decrease in medical costs relative to selexipag. This study provides real-world evidence to suggest that, in patients with $\mathrm{PAH}$, treatment with oral treprostinil may be more cost effective than selexipag over a 6- to 12-month period.

\subsection{Limitations}

ICD-9-CM and ICD-10-CM diagnosis codes, the standard method for identifying patients in claims studies, can be used to identify PH but are not specific to the subclassification of patients with PAH. These diagnosis codes used for billing and administrative purposes do not align with the WHO clinical classification scheme, which classifies PH into groups of conditions that share similar underlying etiologies and are used by $\mathrm{PH}$ specialists to diagnose and medically manage PH [25, 31]. Thus, for this study, PAH was identified using previously developed algorithms for identifying patients with PAH from claims databases [25, 31] by combining requirements for a $\mathrm{PH}$ diagnosis and a claim for a PAH-indicated medication (i.e., an oral prostacyclin). Using diagnosis and pharmacy claims can be imprecise, and some patients may have been misclassified. However, since prostacyclin therapies are only indicated for the treatment of PAH, and most healthcare payers/insurers require a confirmed PAH diagnosis prior to authorization, the combination of a diagnosis and a PAH-indicated prostacyclin medication is likely to reduce the probability of a patient without PAH being included in the study cohort.

The study was conducted in a US managed care population, and PAH is a rare disease, thus the sample size was relatively small, so caution needs to be used while generalizing these results to other PAH populations. Some of the outcomes reported in this study (e.g., inpatient hospitalizations) may be more appropriate for longer follow-up than the 6 months reported herein. However, when continuous enrollment for 12 months was evaluated, small sample sizes resulted because of PAH being a rare disease, the relatively small market share given the recency of oral prostacyclin product launches, health insurance plan changes, and deaths. Thus, a sensitivity analysis of outcomes at 12 months was evaluated when possible. Patients in the oral treprostinil 
cohort appeared slightly sicker at baseline, with higher HCRU. However, PAH disease severity cannot be ascertained using claims data, which do not include clinical information such as pulmonary pressure, FC, or 6-minute walk test results. Thus, PAH disease severity could not be directly controlled for in the adjusted analyses. While prescription claims were available, we were unable to determine whether medication was dispensed and taken as prescribed. While this could result in changes to the utilization and costs of care, assessment of prescription days of supply indicated that patients with PAH receiving oral prostacyclin therapies in the study had high medication adherence. Lastly, claims data do not contain information on dose changes. Thus, understanding of the actual doses taken by patients, including changes during dosing titration, is challenging in claims data.

\section{Conclusions}

This is the first real-world study to compare healthcare utilization and costs between patients treated with either oral treprostinil or selexipag. In this real-world setting, the demographic and clinical characteristics of patients receiving oral treprostinil and selexipag for the treatment of PAH did not differ appreciably. Adherence and persistence were similar, although the mean MPR was slightly higher for patients receiving oral treprostinil. Few differences were seen in nonpharmacy-related utilization and costs between the oral treprostinil and selexipag cohorts, but lower pharmacy costs for oral treprostinil resulted in significantly lower total healthcare costs than for patients receiving selexipag.

Although a sensitivity analysis was conducted to examine HCRU over a 12-month follow-up, it will be important to look at longer-term utilization and cost outcomes among patients initiating oral prostacyclins in a larger population and to explore differences in clinical outcomes and mortality.

Author Contributions Dr. Dean, Mr. Saundankar, Dr. Stafkey-Mailey, Dr. Anguiano, Dr. Nelsen, Dr. Gordon, and Mr. Classi have provided substantial contributions to the conception or design of the work and analysis and interpretation of the data. Dr. Dean and Mr. Saundankar drafted the work and Dr. Stafkey-Mailey, Dr. Anguiano, Dr. Nelsen, Dr. Gordon, and Mr. Classi reviewed it critically for important intellectual content. All authors have provided final approval of the manuscript to be published and agree to be accountable for all aspects of the work in ensuring that questions related to the accuracy or integrity of any part of the work are appropriately investigated and resolved.

\section{Compliance with Ethical Standards}

Funding This research was funded by United Therapeutics Corporation.
Conflicts of interest Dr. Dean, Mr. Saundankar, and Dr. Stafkey-Mailey are employees of Xcenda, LLC, which received financial support for the conduct of this study. Dr. Anguiano has received personal fees from United Therapeutics Corporation, outside the submitted work. Dr. Nelsen, Dr. Gordon, and Mr. Classi are employees of United Therapeutics Corporation, manufacturer of oral treprostinil.

Data availability The data that support the findings of this study are available from IBM ${ }^{\circledR}$, but restrictions apply to the availability of these data, which were used under license for the current study, and so are not publicly available. However, data are available from the authors upon reasonable request and with permission of IBM $^{\circledR}$.

Open Access This article is licensed under a Creative Commons Attribution-NonCommercial 4.0 International License, which permits any non-commercial use, sharing, adaptation, distribution and reproduction in any medium or format, as long as you give appropriate credit to the original author(s) and the source, provide a link to the Creative Commons licence, and indicate if changes were made. The images or other third party material in this article are included in the article's Creative Commons licence, unless indicated otherwise in a credit line to the material. If material is not included in the article's Creative Commons licence and your intended use is not permitted by statutory regulation or exceeds the permitted use, you will need to obtain permission directly from the copyright holder.To view a copy of this licence, visit http://creativecommons.org/licenses/by-nc/4.0/.

\section{References}

1. Galiè N, Humbert M, Vachiery JL, et al. 2015 ESC/ERS guidelines for the diagnosis and treatment of pulmonary hypertension: The Joint Task Force for the Diagnosis and Treatment of Pulmonary Hypertension of the European Society of Cardiology (ESC) and the European Respiratory Society (ERS). Eur Heart J. 2016;1;37(1):67-119.

2. Thenappan T, Shah SJ, Rich S, Tian L. Survival in pulmonary arterial hypertension: a reappraisal of the NIH risk stratification equation. Eur Respir J. 2010;35:1079-87.

3. Gall H, Felix JF, Schneck FK, et al. The Giessen Pulmonary Hypertension Registry: survival in pulmonary hypertension subgroups. J Heart Lung Transplant. 2017;36:957-67.

4. Benza RL, Miller DP, Barst RJ, et al. An evaluation of long-term survival from time of diagnosis in pulmonary arterial hypertension from the REVEAL Registry. Chest. 2012;142(2):448-56.

5. Farber HW, Miller DP, Poms AD, et al. Five-year outcomes of patients enrolled in the REVEAL Registry. Chest. 2015;148(4):1043-54.

6. Benza R, Gomberg-Maitland M, Elliot G, et al. Predicting survival in patients with pulmonary arterial hypertension: the REVEAL risk score calculator 2.0 and comparison with ESC/ERS-based risk assessment strategies Chest. 2019;156(2):323-37.

7. Hoeper MM, Kramer T, Pan Z, et al. Mortality in pulmonary arterial hypertension: prediction by the 2015 European pulmonary hypertension guidelines risk stratification model. Eur Respir J. 2017;50(2):1700740.

8. Boucly A, Weatherald J, Savale L, et al. Risk assessment, prognosis and guideline implementation in pulmonary arterial hypertension. Eur Respir J. 2017;50(2):1700889.

9. Kylhammar D, Kjellström B, Hjalmarsson C, et al. A comprehensive risk stratification at early follow-up determines prognosis in pulmonary arterial hypertension. Eur Heart J. 2018;14;39(47):4175-81. 
10. Dufour R, Pruett J, Hu N, et al. Healthcare resource utilization and costs for patients with pulmonary arterial hypertension: real-world documentation of functional class. J Med Econ. 2017;20(11):1178-86.

11. Casserly B, Klinger JR. Brain natriuretic peptide in pulmonary arterial hypertension: biomarker and potential therapeutic agent. Drug Des Devel Ther. 2009;3:269-87.

12. Galiè N, Corris PA, Frost A, et al. Updated treatment algorithm of pulmonary arterial hypertension. J Am Coll Cardiol. 2013;62(25 Suppl):D60-72.

13. Galiè N, Barberà JA, Frost AE, et al. Initial use of ambrisentan plus tadalafil in pulmonary arterial hypertension. N Engl J Med. 2015;373:834-44.

14. Sitbon O, Ja1s X, Savale L, et al. Upfront triple combination therapy in pulmonary arterial hypertension: a pilot study. Eur Respir J. 2014;43:1691-7.

15. Klinger JR, Elliott CJ, Levine DJ, et al. Therapy for pulmonary arterial hypertension in adults: update of the CHEST guideline and expert panel report. Chest. 2019;155(3):565-86.

16. Ruan C-H, Dixon RAF, Willerson JT, Ruan K-H. Prostacyclin therapy for pulmonary arterial hypertension. Tex Heart Inst. 2010;37(4):391-9.

17. Studer S, Hull M, Pruett J, et al. Treatment patterns, healthcare resource utilization, and healthcare costs among patients with pulmonary arterial hypertension in a real-world US database. Pulm Circ. 2018;9(1):1-12.

18. Burger C, Ozbay A, Lazarus H, et al. Treatment patterns and associated health care costs before and after treatment initiation among pulmonary arterial hypertension patients in the United States. J Manag Care Spec Pharm. 2018;13:1-9.

19. Burger C, Pruett J, Lickert C, Berger A, Murphy B, Drake W. Prostacyclin use among patients with pulmonary arterial hypertension in the United States: a retrospective analysis of a large health care claims database. J Manag Care Spec Pharm. 2018;24(3):291-302.

20. Tapson VF, Torres F, Kermeen F, et al. Oral treprostinil for the treatment of pulmonary arterial hypertension in patients on background endothelin receptor antagonist and/or phosphodiesterase type 5 inhibitor therapy (the FREEDOM-C study). Chest. 2012;142(6):1383-8.
21. Sitbon O, Channick R, Chin KM, et al. Selexipag for the treatment of pulmonary arterial hypertension. N Engl J Med. 2015;373:2522-33.

22. Coghlan JG, Channick R, Chin K, et al. Targeting the prostacyclin pathway with selexipag in patients with pulmonary arterial hypertension receiving double combination therapy: insights from the randomized controlled GRIPHON study. Am J Cardiovasc Drugs. 2018;18:37-47.

23. Tapson VF, Sanchez-Diaz CJ, Bohns-Myers GM, et al. Treatment with oral treprostinil delays time to clinical worsening in patients with pulmonary arterial hypertension - results from FREEDOMEV. J Heart Lung Transpl. 2019;38(4 suppl):S94-5.

24. White et al. Combination therapy with oral treprostinil for pulmonary arterial hypertension: a double-blind, placebo-controlled study. Am J Respir Crit Care Med. 2019. https://doi.org/10.1164/ rccm.201908-1640oc (Epub ahead of print).

25. Mathai SC, Hemnes AR, Manaker S, et al. Identifying patients with pulmonary arterial hypertension using administrative claims algorithms. Ann Am Thorac Soc. 2019;16(7):797-806.

26. Kleinman LC, Norton EC. What's the risk? A simple approach for estimating adjusted risk measures from nonlinear models including logistic regression. Health Serv Res. 2009;44(1):288-302.

27. Zhongmin L, Maendra G. Using "recycled predictions" for computing marginal effects. SAS Global Forum 2010. https:// support.sas.com/resources/papers/proceedings10/272-2010.pdf. Accessed 29 Jan 2020.

28. Basu A, Polsky D, Manning WG. Estimating treatment effects on healthcare costs under exogeneity: Is there a 'magic bullet'? Health Serv Outcomes Res Methodol. 2011;11(1-2):1-26.

29. Lexchin J. Pricing of multiple dosage prescription medications: an analysis of the Ontario drug benefit formulary. Health Policy. 2009;91(2):142-7.

30. Truong J, Chan KKW, Mai H, et al. The impact of pricing strategy on the costs of oral anti-cancer drugs. Cancer Med. 2019;8(8):3770-81.

31. Gillmeyer KR, Lee MM, Link AP, et al. Accuracy of algorithms to identify pulmonary arterial hypertension in administrative data a systematic review. Chest. 2019;155(4):680-8. 